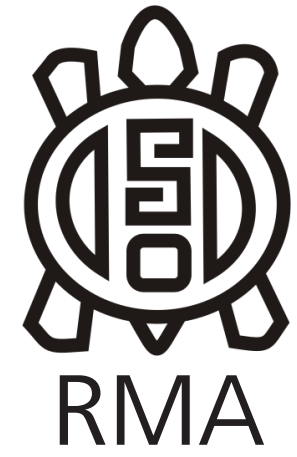

\title{
Perspectiva de Análisis Integral de Artefactos Líticos: Casos de Bifaces del Sitio Quebrada Seca 3 (Antofagasta de la Sierra, Catamarca)
}

\author{
A Perspective of Integral Analysis for the Study of Stone Tools: Cases of \\ Bifaces of Quebrada Seca 3 Site (Antofagasta de la Sierra, Catamarca)
}

Salomón Hocsman* y Pilar Babot**

\begin{abstract}
Dossier
* Instituto Superior de Estudios Sociales, CONICET-Universidad Nacional de Tucumán Instituto de Arqueología y Museo, Facultad de Ciencias Naturales e Instituto Miguel Lillo, Universidad Nacional de Tucumán, Argentina. E-mail: shocsman@hotmail.com ** Instituto Superior de Estudios Sociales, CONICET-Universidad Nacional de Tucumán - Instituto de Arqueología y Museo, Facultad de Ciencias Naturales e Instituto Miguel Lillo, Universidad Nacional de Tucumán, Argentina.

E-mail: pilarbabot@csnat.unt.edu.ar
\end{abstract}

\begin{abstract}
Resumen
En este trabajo se exponen los parámetros y una serie de consideraciones sobre lo que se denomina perspectiva integral en el análisis de artefactos líticos, que implica un estudio situado o situacional (y coyuntural) de sus historias de vida mediante una complementariedad de líneas carentes de relación jerárquica que se interpelan y dialogan entre sí, en un abordaje no destructivo o microdestructivo (de bajo impacto) y plausible de implementarse en materiales de colección, donde la información contextual es relevante. Luego, se presentan casos seleccionados de bifaces del sitio Quebrada Seca 3 (Antofagasta de la Sierra, Catamarca), datados entre 5500 y 4500 años AP. Estos artefactos se analizan desde una perspectiva que integra de manera complementaria y equivalente los datos originados en múltiples líneas de evidencia: estudio tecno-tipológico de detalle, análisis múltiple de microfósiles por microscopía de polarización, análisis lipídicos mediante Cromatografía Gaseosa-Espectrometría de Masas (GC-MS), de adhesivos empleados en el enmangue a través de Espectrometría de Infrarrojos por Transformada de Fourier (FTIR) e información contextual de los niveles considerados.
\end{abstract}

Palabras clave: Perspectiva de análisis integral; Múltiples líneas de evidencia; Artefactos líticos; Bifaces; Puna Argentina.

\begin{abstract}
In this work the parameters and a series of issues on what is called integral perspective in the analysis of lithic artifacts, are exposed. This perspective implies a situated or situational (and conjunctural) study of their life stories through a complementarity of lacking of hierarchy lines which interpellate and dialogue with each other, within a non-destructive or micro-destructive (low impact) approach that is plausible to be implemented in museum materials, in which contextual information is relevant. Then, some bifaces from the Quebrada Seca 3 site (Antofagasta de la Sierra, Catamarca) dating from 5500-4500 years BP, are displayed as selected cases. These artifacts are analyzed from a perspective that integrates multiple complementary and equivalent lines of evidence, namely: detailed technotypological study, multiple microfossil analysis by polarization microscopy, lipid analysis by Gas Chromatography-Mass Spectrometry (GC-MS), analysis of adhesives used in the hafting through Fourier Transform Infrared Spectrometry (FTIR), and contextual information from the layers considered.
\end{abstract}

Keywords: Integral of perspective análisis; Multiple lines of evidence; Stone tools; Bifaces; Argentinean Puna.

\section{Introducción}

La identificación y caracterización de los usos de los artefactos líticos es un tema que ha gozado de un profundo interés en Argentina, en el que interesa la instrumentación de múltiples líneas de evidencia. Si bien los estudios dominantes combinaron análisis tecno-tipológicos y funcionales de base microscópica -microrrastros- (Manzur 1986; Landini et al. 2000; Álvarez 2009; Cattáneo y Aguerre 2009; Massigoge y Pal 2011; Leipus 2016; entre otros), con el desarrollo y aplicación de nuevas técnicas y metodologías se diversificaron las líneas de evidencia empleadas y las preguntas se orientaron a la historia de vida artefactual (Babot 2010; Babot et al. 2013; Escola et al. 2013; Mazzia et al. 2016; Cattáneo et al. 2017).

En este trabajo se desarrollan aspectos teóricometodológicos relevantes para un estudio de este tipo a partir de la experiencia generada desde este último enfoque de la perspectiva integral de análisis en Antofagasta de la 
Sierra (Catamarca), donde se pueden citar abordajes de: a) los artefactos de molienda formatizados y modificados por uso de diferentes momentos del Holoceno, conjugando análisis tecno-tipológico, de microfósiles, de residuos orgánicos e inorgánicos, contextual y de saberes tradicionales (Babot 1999, 2004); b) las puntas de proyectil/ cuchillos enmangados de los niveles aquí considerados de QS3, desde las líneas tecno-tipológica, huellas de uso, microfósiles, adhesivos para enmangue/enastilado, análisis de residuos lipídicos y contextual del sitio (Babot et al. 2010; Babot et al. 2013); y c) los cuchillos/raederas de módulo grandísimo de contextos agro-pastoriles plenos, desde las líneas tecno-tipológica, de microfósiles, de análisis de residuos lipídicos, de desechos de reactivación, contextual de los sitios, de las prácticas tradicionales, del registro macro arqueobotánico y macro y microentomológico (Babot et al. 2008; Escola et al. 2013).

Luego, se presentan casos destacados de bifaces recuperados en el sitio Quebrada Seca 3 (QS3), localizado en Antofagasta de la Sierra (Puna de Catamarca), para ilustrar la perspectiva de estudio integral. QS3 es un abrigo rocoso ubicado al pie del farallón de ignimbritas que conforma la margen sur de la vega de Quebrada Seca, a $4100 \mathrm{msnm}$. Presenta un área de reparo a modo de alero (exterior) y cueva (interior) cuyas dimensiones lineales, en ancho y profundidad desde el área de goteo hasta la pared del fondo, son de 9 × 5m (Aschero et al. 1993-94). Las piezas consideradas proceden de las capas superiores del sitio (2b2 a 2b5), datadas entre los 5500 y los 4500 años $A P$, correspondientes a cazadores-recolectores finales, en un momento inicial de la domesticación animal y del manejo de plantas domesticadas. Los contextos fueron asignados a una base residencial con espacios acondicionados y equipamiento, ocupada estacionalmente, pero de manera frecuente o recurrente por grupos humanos de pequeño tamaño. El carácter de las ocupaciones se ha establecidosegún evidencias proporcionadas por la organización del espacio y las actividades, el descarte/abandono del material lítico y faunístico, la ubicación de fogones y el área de dispersión carbonosa en torno a ellos, la disposición de camadas de paja, etc., definiendo una alta estructuración del espacio intra-sitio, recurrente a lo largo de aproximadamente mil años (Aschero et al. 1991, 1993-94). Entre las actividades registradas se encuentran el procesamiento vegetal y animal para consumo y la manufactura y uso de instrumentos en fibras vegetales y piedra (Babot 2004; Rodríguez 2013; entre otros). Se destaca en particular un locus de preparación y recambio de cabezales líticos en torno a estructuras de combustión. Una parte de estos instrumentos, los denominados puntas/cuchillo han sido objeto de un estudio integral multiproxy anterior (Babot et al. 2013), así como el instrumental de molienda del sitio (Babot 2004). Estos antecedentes y demás información contextual dialogan en la interpretación que proponemos acerca de la historia de vida de los bifaces.
Los bifaces se analizan aquí a partir de una propuesta que combina y complementa los abordajes morfológicofuncional, técnico-morfológico, dimensional y funcional desde diferentes propuestas analíticas de la arqueobotánica y la físicoquímica, tendientes a evaluar los diferentes roles cumplidos por estos artefactos a lo largo de historias de vida variables. De esta forma, interesa distinguir entre bifaces como instrumentos, como soporte de instrumentos, en proceso de manufactura y como núcleos, entre otras posibilidades (Aschero y Hocsman 2004). A esto se suma la destreza técnica diferencial como un elemento a considerar (Hocsman 2007). Finalmente, la indagación en su conjunto se orienta a obtener una mejor comprensión de las prácticas sociales a las que las piezas se integraron.

\section{La Perspectiva de Análisis Integral Propuesta}

La perspectiva de análisis integral de artefactos líticos implica la integración de diferentes líneas de evidencia para establecer aspectos diversos de la producción y utilización que nos aproximen a la historia de vida de una determinada clase o conjunto artefactual y, a un nivel mayor, para contribuir a la caracterización de las prácticas referidas al lugar, a las plantas y los animales, entre otros, sentido último del estudio de casos.

En la Figura 1 se representa un mapa conceptual en el que destacan tres líneas analíticas principales para el caso de los artefactos líticos y sus vinculaciones con el contexto de origen y su historia de formación para dar cuenta de la historia de vida de los utensilios.

Ahora bien, la perspectiva integral no es una simple sumatoria de técnicas analíticas, sino un estudio que es situado o situacional y, por lo tanto, funcional al caso y a la pregunta por detrás. También es coyuntural porque hay un grado de dependencia de la disponibilidad y accesibilidad de las técnicas, equipos, expertos y de su conocimiento específico del tema que son identificados como la situación ideal para el caso que se aborda. Finalmente, es deseable que se desarrolle como una colaboración ad hoc y, por lo tanto, en el corto plazo. Sin embargo, el estudio integral se puede ir completando en el tiempo, en cuyo caso, los resultados parciales tienen su propio valor, aunque el momento de la síntesis será definitorio.

Entonces, el estudio es situado o situacional porque se requiere de un diseño específico y de la elección de las técnicas más apropiadas en cada caso. Estas elecciones también están condicionadas por el grado de definición u orientación de las hipótesis iniciales. Por ejemplo, en el caso de los bifaces, a diferentes escalas, se esperaría: a) un registro de uso versus su ausencia (núcleos bifaciales o bifaces de aprendices); b) un registro de uso sobre materiales de origen animal o vegetal o mineral o indistinto; y c) un registro de uso referido al destazamiento de fauna o al corte de plantas leñosas. 
De este modo, lo importante no es la cantidad de líneas y técnicas instrumentadas, aunque a mayor cantidad de líneas la aproximación es más completa o autocomprobable, sino la pertinencia y adecuación al caso y la forma de implementarlas, destacando el concepto de complementariedad de la información obtenida. Estas líneas analíticas se interpelan y dialogan entre sí a lo largo de toda la investigación.

Ahora bien, se sostiene que no existe una relación jerárquica entre las líneas que se integran. En cambio, es preciso tener presentes las debilidades y fortalezas de cada aproximación y el tipo de dato, grano o detalle que se puede obtener de ellas. Esto es importante, por ejemplo, durante la valoración de los resultados positivos y negativos, en particular, cuando aparentan no ser concordantes luego de haber seguido procesos independientes en ciego. Este diálogo es el que va a permitir proponer si es posible sostener una situación de uso, ausencia de uso, contaminación, pérdida post-depositacional de la evidencia de uso, etc., dependiendo del caso.

Así, uno de los aspectos en los que la perspectiva integral es superadora del estudio de líneas independientes es la posibilidad de proponer la ocurrencia de negativos metodológicos para determinadas técnicas, es decir, situaciones de invisibilidad del registro para una técnica y, por contrapartida, su visualización mediante otras técnicas. Esto impactará por ejemplo en la posibilidad de realizar un movimiento desde una interpretación a priori en términos de contaminación o modificación post-depositacional del registro que puede ser descalificante de los resultados de otras líneas, a otra que permita ganar en la comprensión del alcance de cada línea por separado, en donde el dato post-depositacional se integra positivamente a la historia artefactual. Metodológicamente, estas situaciones permiten dar un paso más allá, por ejemplo, cuando muestran la pertinencia de ampliar las colecciones de referencia contra las que se comparan los datos arqueológicos, haciendo que el campo analítico se expanda y fortalezca. Ejemplos pueden ser: a) en una situación adversa para la preservación de residuos biológicos, el registro del desgaste por uso puede ser determinante; b) en una situación del registro de trabajo sobre un material poco experimentado en el ámbito del desgaste por uso, como las tuberosas, el registro de microfósiles, en particular, del almidón, será determinante.

Otro aspecto que interesa destacar es que todo abordaje integral debería tender a ser no destructivo o microdestructivo (o ser de bajo impacto) por tres motivos: a) la preservación o conservación de los bienes patrimoniales, b) posibilitar la intervención de múltiples analistas con el menor impacto posible sobre la materia y sus propiedades entre las sucesivas fases de análisis y c) la replicabilidad de los experimentos. Esta consideración operará en el orden en que los sucesivos analistas intervengan sobre las piezas, en la demanda de la recuperación de las submuestras que son empleadas en distintas técnicas para su reciclaje y la manipulación responsable del material desde el momento cero de la exhumación en campo, siempre que es posible: rápido embalado; selección adecuada del material de embalaje; muestreo de control de la matriz de depósito; preservación de las muestras en condiciones apropiadas de humedad, temperatura e iluminación; manipulación con guantes libres de polvo y barbijo; recuperación y reserva de los subproductos de la limpieza; recuperación

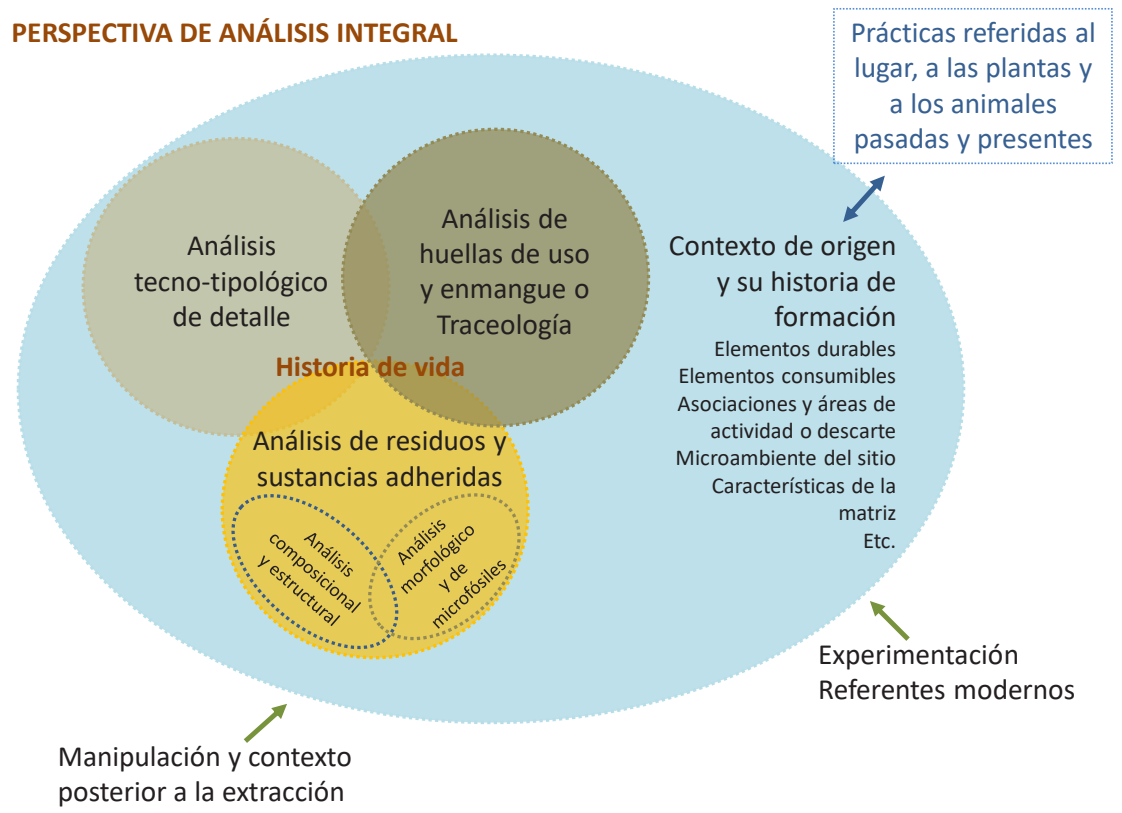

Figura 1. Líneas de análisis e interpretación principales en una perspectiva de estudio integral de artefactos líticos.

Figure 1. Main lines of analysis and interpretation in a perspective of integral study of lithic artifacts. 
de las submuestras; reciclaje o reuso de las mismas; y registro gráfico y escrito de las intervenciones.

De igual modo, también es importante el estudio de material de colecciones en donde no todos estos recaudos han sido posibles. Esto puede cobrar un valor particular en los casos de piezas o conjuntos de piezas únicas, o que proceden de contextos especiales o únicos, contextos que no son accesibles al momento del análisis, que son inexistentes al presente por distintos motivos, u objetos que han sido hallados en circunstancias no previstas de un futuro análisis funcional. El estudio de colecciones también puede ser un objetivo de análisis integral en el caso de piezas que proceden de contextos que se conocen muy bien, para los cuales hay mucha información y que puedan ya haber sido sujetos de otros análisis de tipo integral.

En una perspectiva de análisis integral la información contextual es clave porque permite considerar numerosos aspectos. Por ejemplo, ¿estarían dadas las condiciones microambientales para la preservación de residuos orgánicos?, ¿cabría esperar un registro de meteorización?, ¿sería factible la contaminación cruzada desde el contexto?, ¿se trata de una asociación primaria o secundaria?, ¿qué se esperaría de la historia de vida del artefacto dado el conocimiento ya existente sobre el contexto de origen y su historia de formación? Además, dado que el estudio es situado, la vuelta al contexto post-analítica es central.

Finalmente, destaca la importancia de las colecciones de referencia y de bases de datos asociadas que sean continuamente mejoradas y re-visitadas, en función de aspectos resultantes de las investigaciones en curso o, sencillamente, del incremento progresivo de dichas colecciones. Igualmente relevantes son otros referentes contemporáneos cuando están disponibles, como la información referida a los saberes tecnológicos actuales y subactuales y la experimentación, que constituyen insumos de hipótesis para toda la investigación o para alguna de las líneas en particular y, a la vez, intervienen en la fase interpretativa de los datos.

\section{Casos de Bifaces de Quebrada Seca 3}

\section{Metodología}

Los bifaces son considerados como artefactos que se caracterizan por sus múltiples formas de presentación y posibilidades de uso, ya que pueden referir tanto a instrumentos o a núcleos, como a piezas en proceso de manufactura (Hocsman 2019). Se seleccionan casos relevantes para ilustrar la articulación de diferentes líneas de evidencia que se describen a continuación. a) El estudio tecno-tipológico de detalle siguió las propuestas de Aschero (1975, 1983), Aschero y Hocsman (2004) y Hocsman (2009). b) En el análisis múltiple de microfósiles por microscopía de polarización se siguió un proceso de muestreo total no destructivo mediante el escarbado de un área constante en la superficie rocosa con un instrumento metálico limpio. En las observaciones entre 100 y $630 X$ se utilizó un microscopio petrográfico Leica DM750P con cámara digital acoplada registrándose diferentes micropartículas de interés (granos de almidón, silicofitolitos, calcifitolitos, fibras, fragmentos de tejido vegetal desecado, entre otros). Las imágenes obtenidas fueron procesadas a través del software LAS EZ (Leica Application Suite). c) Los análisis lipídicos se llevaron adelante mediante Cromatografía Gaseosa-Espectrometría de Masas (CG-EM), y d) los estudios de adhesivos empleados en el enmangue, a través de Espectrometría de Infrarrojos por Transformada de Fourier (FTIR). A los fines del estudio por CG-EM, las extracciones sólido-líquido con cloroformo se realizaron en un baño ultrasónico, sumergiendo durante un minuto los instrumentos. Estos extractos se filtraron en papel de filtro de $45 \mu \mathrm{m}$ lavados previamente con cloroformo, durante el tiempo necesario para obtener muestras libres de sedimentos, y se secaron con nitrógeno. Los extractos se escanearon en un sistema GC Hewlett Packard HP 6890 Series acoplado a un detector selectivo de masas Hewlett Packard 5973, utilizando el método de escaneo TTP con un tiempo de ejecución entre 0-60 'y 0-30'. Estos se condujeron en el Instituto de Química Orgánica, Facultad de Bioquímica, Química y Farmacia, Universidad Nacional de Tucumán. Los análisis de FTIR se realizaron en la Comisión Nacional de Energía Atómica. Una muestra pequeña de adhesivo se dispuso directamente en el portamuestras, escaneándola entre 650 y $4000 \mathrm{~cm}^{-1}$ en modo Reflactancia. e) Finalmente, la interpretación integral de los datos se desarrolló considerando la información contextual de los niveles considerados, casos de análisis integrales precedentes en el sitio e información adicional del área de estudio para las líneas de evidencia aquí consideradas.

Se trata de un conjunto de piezas de colección, recuperadas décadas atrás, pero conservadas en sus bolsas plásticas individuales de origen. Debido a esta situación, no fue posible realizar una muestra de control del contenido lipídico del sedimento de la matriz. No obstante, los resultados de esta línea (posibilidades de contaminación cruzada in situ o postexcavación) se evaluaron al cotejarlos con los datos de análisis similares realizados en artefactos de los mismos niveles por CGEM y FTIR (Tabla 1; Figura 2) y con la información de macro y microrrestos vegetales ya conocida para el sitio y el área de estudio en general (Babot 2004; Rodríguez 2013; entre otros). Asimismo, se cotejó la coherencia de los resultados con las expectativas iniciales y con los datos proporcionados por las restantes líneas analíticas. Dos factores considerados como testigos de alteraciones postdepositacionales en varios sitios del área, son la habitación subactual y potencial cohabitación pasada de aves y la colonización por líquenes. Por ello se relevó mediante CG-EM el contenido de las heces de estos animales y de líquenes secos (Tabla 1). De igual modo, se controlaron las señales de plastificantes (ftalatos) y otras sustancias industriales presentes en las bolsas y solventes. 
A continuación, se presentan los casos analizados, introduciendo en cada uno de ellos las hipótesis de uso derivadas del análisis tecno-tipológico y finalizando con una breve interpretación sobre la vida útil del artefacto que apela a la información contextual y areal.

\section{Expectativas, Resultados y Discusión de los casos analizados}

Desde el análisis tecno-tipológico el artefacto $N^{\circ} 65$ se define como un fragmento meso-basal de pieza enmangada sobre bifaz parcial en el que se aprecia la porción proximal de un limbo fracturado que posee un cuchillo microdenticulado en un borde y un filo bifacial de arista sinuosa en el otro borde con una amplitud del sinusoide muy estrecha y un bisel agudo. Presenta un sector basal conformado por tres filos bifaciales de arista sinuosa, menos estrechos y más abruptos, que definen dos laterales y una base. Se observan dos puntos de inflexión claros entre los bordes del sector basal y los bordes del limbo y residuos en sus caras. Estas características permiten calificarlo como un bifaz como instrumento enmangado (Hocsman 2019), de lo que se desprenden como expectativas: la verificación de un uso efectivo en el limbo y de evidencias de enmangue, incluyendo adhesivos en el sector basal. Lo último se estableció mediante la caracterización microscópica del residuo rojizo basal in situ y por FT-IR que indican un adhesivo natural compatible con resinas terpénicas (Bruni y Guglielmi 2014) (Figura 2). Dirigiendo la mirada al contexto estratigráfico, se aprecian diferentes indicadores ligados al enmangue y desenmangue de los cabezales líticos en torno a estructuras de combustión en las capas consideradas, dados por fragmentos de puntas-cuchillos, piezas enteras, un extremo proximal de intermediario en caña Chusquea lorentziana que contenía adhesivos adheridos, más un tendón de un diámetro similar atado al mismo (Aschero et al. 1991; Aschero et al. 1993-94). También se verifica una correspondencia entre el adhesivo registrado en el bifaz $\mathrm{N}^{\circ}$ 65 con el que presentan cinco puntas/cuchillo recuperadas en las capas $2 \mathrm{~b}(3)-\mathrm{N}^{\circ} 510-, 2 \mathrm{~b}(4)-\mathrm{N}^{\circ} 109-$ y $2 \mathrm{~b}(5)-\mathrm{N}^{\circ} 67$, 68 y 69-(Babot et al. 2013) (Figura 2), aportando así a las circunstancias de producción y mantenimiento de este instrumento en un conjunto artefactual mayor.

La pieza $N^{\circ} 28$ corresponde a un fragmento mesobasal de bifaz en sentido estricto (Figura $3 A-C$ ). Cuenta con un filo bifacial de arista sinuosa estrecho y agudo. No presenta evidencias morfológicas macroscópicas vinculadas a enmangue. Por esto, la prehensión sería de tipo manual, definiéndose un bifaz como instrumento de prehensión manual. Así, se estima la ausencia de evidencias de enmangue, que se corrobora desde la falta de residuos macroscópicos y microscópicos de adhesivos. La expectativa de un uso efectivo en el limbo se verifica mediante el análisis de residuos lipídicos por CG-EM que arroja un conjunto de ácidos grasos (Ác. mirístico, palmítico y esteárico) relativamente comunes en la naturaleza en fuentes vegetales y animales y de cierta ubicuidad en las

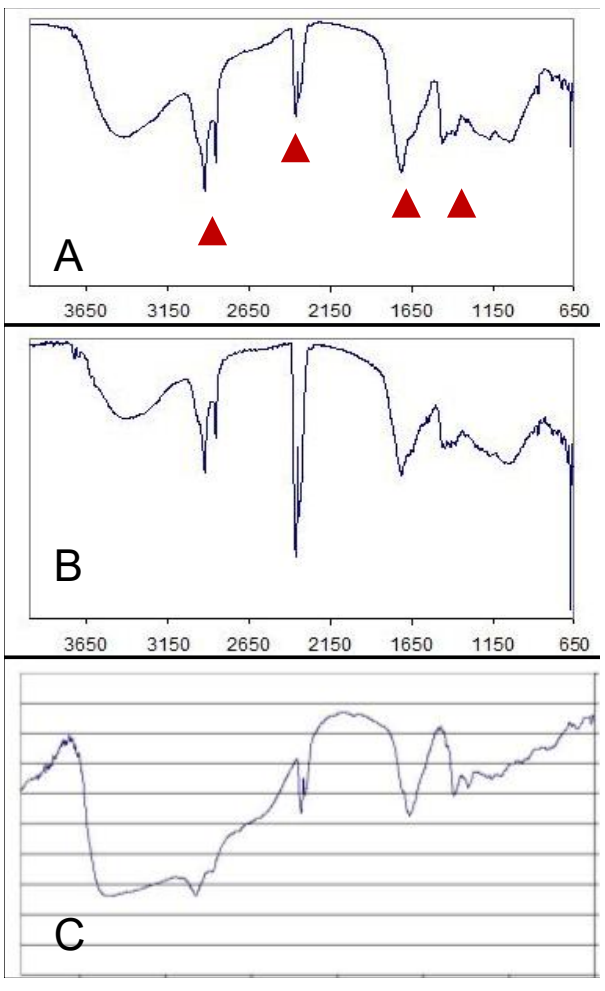

Figura 2. Espectros FT-IR (Reflectancia) entre 650 y $4000 \mathrm{~cm}^{-1}$; se señalan las bandas de interés. A. Sustancia resinosa rojiza en zona de enmangue del bifaz № 65 -nivel $2 b(5)$ de QS3. B. Sustancia resinosa en zona de enmangue de la punta/ cuchillo N67 de QS3 -nivel 2b(5). C. Resina de yareta (Azorella compacta Phil. Apiaceae).

Figure 2. Spectra FT-IR (Reflectance) between 650 and $4000 \mathrm{~cm}$ 1 ; the bands of interest are indicated. $A$. Reddish resinous substance in shafting area of biface $N^{\circ} 65$-level $2 b(5)$. B. Resinous substance in shafting area of projectile point / knife $N^{\circ} 67$ of QS3 -level 2b (5). C. Yareta resin (Azorella compact Phil. Apiaceae).

muestras de distinta cronología del área de estudio, por lo que su diagnosis es reducida (Tabla 1). Destacan otros compuestos más indicativos del material trabajado. La presencia de escualeno puede indicar el procesamiento de una sustancia animal y, al mismo tiempo, proceder de la propia manipulación humana por tratarse de una pieza lítica de uso manual. Es un triterpeno abundante en la piel que solo integra minoritariamente aceites vegetales (Sánchez Vizcaíno y Cañabate Guerrero 1998). Su registro en el área de estudio se circunscribe, además, a una mano de moler y a un cuchillo-raedera de sitios agro-pastoriles (Tabla 1). En estos dos casos, la presencia de este compuesto podría vincularse con el procesamiento de plantas cultivadas de la familia Amaranthaceae (Escola et al. 2013) además de las alternativas propuestas para el bifaz. Se descarta la contaminación durante la manipulación de laboratorio por haber seguido el mismo procedimiento en todas las muestras que han resultado positivas o negativas para este compuesto.

Al igual que el escualeno, el ácido benzoico destaca en este 
caso por no ser común en contextos del área de estudio. Únicamente ha sido detectado en residuos de un cuchilloraedera de ocupaciones agro-pastoriles de Antofagasta de la Sierra (Escola et al. 2013). Por este motivo, su registro se estima directamente asociado al uso o la manipulación de estos instrumentos. De particular interés es que éste abunda en la corteza y hojas de especies del género Salix, incluyendo a la única especie nativa del hemisferio sur, $S$. humboldtiana Willd. (Salicaceae) o sauce criollo (Larrahondo y Preston 1989). Esta es una de las cinco especies de plantas leñosas foráneas halladas como macrorrestos vegetales en sitios del área, incluyendo el propio QS3, para una amplia cronología, habiendo sido empleada en astiles (Rodríguez 2013). El hallazgo de cuerpos cristalinos de origen vegetal (drusas y rosetas de 15-40 $\mu$ m y cristales prismáticos de 10$30 \mu \mathrm{m}$ de oxalato de calcio) es afín a este resultado, pues en su conjunto estos microfósiles indican de manera global leños, cortezas y cáscaras (Figura 3D-E). Ambas clases se encuentran, asimismo, en la corteza interna y externa de Salix, junto a tricomas unicelulares eglandulares de 50-300 $\mu \mathrm{m}$ (Duarte y Chella 2013). Un análisis preliminar de rastros de uso del bifaz No 28 (R. Cattáneo com. pers. 2019) sugiere que esta pieza presenta evidencias de utilización efectiva.

Estos resultados permiten también evaluar la integridad del contexto y los residuos en este caso y los siguientes. Las ceras, compuestos alquílicos de cadena larga, en su mayoría hidrocarburos saturados como alcanos y, en menor medida, insaturados como alquenos y otros, de gran estabilidad (Sánchez Vizcaíno y Cañabate Guerrero, 1998), son comunes en otros artefactos de procesamiento vegetal en el área, como el instrumental de molienda (Babot 2004), grandes cuchillos-raederas de sitios agro-pastoriles (Escola et al. 2013) y puntas-cuchillo de QS3 (Babot et al. 2013 (Tabla 1). En esos casos, las ceras se asocian a un contenido relativamente mayor de microfósiles de origen vegetal, indicando el procesamiento de plantas por su frecuencia en epidermis de frutos, hojas, tallos y semillas (Sánchez Vizcaíno y Cañabate Guerrero, 1998). De allí que su inexistencia en el bifaz $\mathrm{N}^{\circ} 28$ y demás artefactos aquí estudiados, y la ausencia de granos de almidón y de silicofitolitos de Poáceas, sugiere: la falta de una contaminación cruzada con las gramíneas que conforman camadas de paja en algunos sectores del sitio, y que piezas de la clase de los bifaces no se usaron en el trabajo de estas herbáceas, tubérculos y semillas. La ausencia de ceras y de otros indicadores (colesterol y particularmente sitosterol), permite obviar la contaminación subactual debida a la cohabitación de aves en el sitio y por líquenes (Babot 2004) (Tabla 1).

La pieza № 593 es un bifaz en sentido estricto, entero, que actúa como soporte de instrumentos, ya que presenta 2 cortantes de filo retocado corto y un filo formatizado pasivo, por lo que se define como bifaz como soporte de instrumento (Hocsman 2019) (Figura 3W). En este caso, la información proporcionada por los residuos lipídicos promediados de los limbos (Ác. láurico, mirístico, palmítico, oleico, esteárico, 15-hexadecanolida) es compatible con la expectativa de un uso efectivo (Tabla 1). Son similares a los del caso anterior, destacándose nuevamente el ácido benzoico con un probable origen en la madera de Salix. Los microfósiles recuperados (cristales diversos) nuevamente se alinean con la ocurrencia de fuentes leñosas como maderas, destacándose su afinidad con Salix (Figura 3X-D'). El ácido laúrico es común en plantas, siendo abundante en especies de arecáceas incluido el género Acrocomia (Arecaceae) (Belén Camacho et al. 2005), lo que se destaca aquí por su posible vinculación con la ocurrencia de cordelería (Rodríguez 2013) y micro-restos de la familia en residuos de uso de manos de moler (Babot 2004) en el sitio QS3. De igual modo, la 15-hexadecanolida es un compuesto aromático raro en muestras del área de estudio (Tabla 1), que se ha hallado en algunas resinas naturales exóticas como en Pinus pinaster, el pino resinero. Hasta el momento, únicamente ha sido detectada en los bifaces y en una punta-cuchillo del sitio QS3 (Babot et al. 2013).

El artefacto $N^{\circ} 632$ es un fragmento meso-apical de bifaz en sentido estricto que, por sus características morfológicas, corresponde a un bifaz en proceso de manufactura (Hocsman 2019) (Figura 3G-I). En este caso no se esperaría un uso efectivo, o bien, un uso y vuelta a la manufactura. Aquí, los residuos lipídicos (Ác. mirístico, pentadecanoico, palmítico, oleico, esteárico, 15-hexadecanolida) remiten a un origen genérico en fuentes animales o vegetales, e incluyen al escualeno que asociamos a la manipulación humana (Tabla 1). Está ausente la señal química específica que se atribuyó a Salix, pero sí se verifican formas cristalinas y tricomas que pueden asignarse al taxón (Duarte y Chella 2013) y a maderas y cortezas en general, junto con fibras, tejidos celulósicos y cuerpos birrefringentes indeterminados (Figura 3J-V). La ausencia de uso registrado a nivel de traceología en los limbos (R. Cattáneo com. pers. 2019), sumado al hecho de que la pieza presenta características morfológicas de estar en proceso de manufactura (Hocsman 2019), lleva a considerar un posible origen para las fuentes lipídicas y microrrestos vegetales genéricos -que se mapean de modo similar en todas las piezas-, en el uso de percutores blandos de madera y la manipulación durante la talla y/o en el uso del bifaz y su vuelta a la manufactura. Nuevamente, como en los artefactos $N^{\circ} 28$ y 593, faltan las señales químicas que antes se discutieron (ceras y esteroles) y que indicarían contaminación cruzada, evaluándose positivamente la integridad del residuo.

Finalmente, la pieza $N^{\circ} 316$ es un bifaz parcial, entero, que posee contorno irregular, sección transversal biconvexa marcadamente asimétrica e irregular, arista sinuosa irregular con sinuosidad moderada a amplia, ángulos de filo importantes y razón ancho/espesor relativamente pequeña, por poseer espesor muy importante, y bordes machacados, fracturados y engrosados, con plano de simetría longitudinal, terminaciones quebradas y cambios de ángulo, siguiendo parámetros de Hocsman $(2007,2019)$ 
Tabla 1. Compuestos identificados en los residuos lipídicos de los bifaces de Quebrada Seca 3 -niveles $2 b(2)$ a $2 b$ (5), en otros artefactos líticos del mismo sitio -presentados como abundancias relativas por artefacto- y del área de estudio -presentados como presencia/ausencia en conjuntos de artefactos de la clase artefactual- y en muestras de control. 'Tomado de Babot et al. (2013). ${ }^{2}$ Tomado de Babot (2004). ${ }^{3}$ Tomado de Escola et al. (2013). ${ }^{4}$ Trazas. ${ }^{5}$ Puede englobar uno o más de los compuestos de esta categoría enumerados de manera individual en la tabla u otros no mencionados.

Table 1. Compounds identified in the lipid residues of the Quebrada Seca 3 bifaces - levels $2 b$ (2) to $2 b$ (5), in other lithic artifacts of the same site -presented as relative abundances per artifact, and of the study area -presented as presence / absence in assemblages of the same artifactual type, and in control samples. ${ }^{1}$ Taken from Babot et al. (2013). ${ }^{2}$ Taken from Babot (2004). ${ }^{3}$ Taken from Escola et al. (2013). ${ }^{4}$ Trazas. ${ }^{5}$ It can include one or more of the compounds in this category listed individually in the table or others not mentioned.

\begin{tabular}{|c|c|c|c|c|c|c|c|c|c|c|c|c|}
\hline Compuesto & $\begin{array}{l}\text { Bifaz } \\
\mathrm{N}^{\circ} 28 \\
\%\end{array}$ & $\begin{array}{c}\text { Bifaz } \\
\text { N593 } \\
\%\end{array}$ & $\begin{array}{c}\text { Bifaz } \\
N^{\circ} 632 \\
\%\end{array}$ & $\begin{array}{c}\text { PP/Cch } \\
\text { No}^{0} 283^{1} \\
\%\end{array}$ & $\begin{array}{c}\text { PP/Cch } \\
\mathrm{N}^{\circ} 292^{1} \\
\%\end{array}$ & $\begin{array}{c}\text { PP/Cch } \\
\mathrm{N}^{0} 508^{1} \\
\%\end{array}$ & $\begin{array}{c}\text { PP/Cch } \\
\mathrm{N}^{\circ} 510^{1} \\
\%\end{array}$ & $\begin{array}{c}\text { PP/Cch } \\
\text { N }^{0} 58^{1} \\
\%\end{array}$ & $\begin{array}{c}\text { Heces } \\
\text { aves }^{2} \\
\%\end{array}$ & $\operatorname{Liq}^{2}$ & $\begin{array}{l}\mathrm{Cch} / \mathrm{I} \\
\mathrm{Rd}^{3}\end{array}$ & $\begin{array}{l}\text { Artef. } \\
\text { mol. }\end{array}$ \\
\hline Ác. benzoico (C7) & 7,7 & 7,0 & & & & & & & & & $\mathrm{x}$ & \\
\hline $\begin{array}{l}\text { Ác. pelargónico } \\
\text { (C9:0) }\end{array}$ & & & & & & & & & & & $\mathrm{x}$ & \\
\hline Ác. cáprico (C10:0) & & & & & & & & & & & $\mathrm{X}$ & \\
\hline $\begin{array}{l}\text { Undecil } \\
\text { dodecanoato (C12) }\end{array}$ & & & & & & 47,4 & & & & & & \\
\hline Ác. láurico (C12:0) & & 1,8 & & & 1,4 & & & & & & $\mathrm{X}$ & \\
\hline Tetradecano (C14) & & & & & 0,6 & & & & & & $\mathrm{X}$ & \\
\hline $\begin{array}{l}\text { Ác. 12- } \\
\text { metiltetradecanoic } \\
\text { o }\end{array}$ & & & & & & & & & & & $\mathrm{x}$ & \\
\hline $\begin{array}{l}\text { Ác. mirístico } \\
\text { (C14:0) }\end{array}$ & 13,1 & 11,5 & 14,1 & & 11,7 & & & 10,9 & & & $\bar{X}$ & \\
\hline $\begin{array}{l}\text { Pentadecano } \\
\text { (C15) }\end{array}$ & & & & & & & & & & & $x$ & \\
\hline $\begin{array}{l}\text { Ác. pentadecílico } \\
\text { (C15:0) }\end{array}$ & & & 4,5 & & 7,2 & & & & & & $\mathrm{x}$ & \\
\hline $\begin{array}{l}\text { 1-Hexadecano } \\
\text { (C16) }\end{array}$ & & & & & & & & 6,2 & & & & \\
\hline Hexadecatrienol & & & & & & & & & & & & $X$ \\
\hline $\begin{array}{l}\text { 15-hexadecanolida } \\
\text { (C16) }\end{array}$ & & 8,4 & 9,2 & & 5,0 & & & & & & & \\
\hline $\begin{array}{l}\text { Ac. palmitoleico } \\
\text { (C16:1) }\end{array}$ & & & & & & & & & & & $x$ & \\
\hline $\begin{array}{l}\text { Ác. palmítico } \\
\text { (C16:0) }\end{array}$ & 46,7 & 41,3 & 44,6 & & 49,9 & & & 46,4 & 24,0 & & $x$ & $x$ \\
\hline $\begin{array}{l}\text { Heptadecano } \\
\text { (C17) }\end{array}$ & & & & & & & & & & & $\mathrm{x}$ & \\
\hline $\begin{array}{l}\text { Metilhexadecanoat } \\
\text { o (C17) }\end{array}$ & & & & & & 3,6 & & & & & & \\
\hline $\begin{array}{l}\text { Ȧc. } \\
\text { heptadecanoico o } \\
\text { margárico (C17:0) }\end{array}$ & & & & & 1,7 & & & & & & & \\
\hline $\begin{array}{l}\text { 1-Octadecanol } \\
\text { (C18) }\end{array}$ & & & & & & & 8,7 & & & & & \\
\hline Octadecano (C18) & & & & & 1,1 & 4,0 & & & & & $\mathrm{X}$ & \\
\hline $\begin{array}{l}\text { 1-Octadeceno } \\
\text { (C18) }\end{array}$ & & & & 15,2 & & & & & & & & \\
\hline $\begin{array}{l}\text { Àc. linolénico } \\
\text { (C18:2) }\end{array}$ & & & & & & & & & 48 & & & \\
\hline Ác. oleico (C18:1) & & 4,5 & 4,6 & & 4,7 & & & 3,6 & & & $\mathrm{X}$ & $\mathrm{X}$ \\
\hline $\begin{array}{l}\text { Ȧc. esteárico } \\
\text { (C18:0) }\end{array}$ & 5,4 & 5,5 & 7,6 & 4,6 & 5,0 & & & 7,8 & 10 & & $\mathrm{x}$ & \\
\hline 9-octadecenamida & & & & & & & & & & & $\mathrm{X}$ & \\
\hline Nonadecano (C19) & & & & & & 3,1 & & & & & $\mathrm{X}$ & \\
\hline Eicosano (C20) & & & & & & 2,8 & & & & & $\mathrm{X}$ & \\
\hline $\begin{array}{l}\text { Heneicosano } \\
\text { (C21) }\end{array}$ & & & & & & & & & & & $X$ & \\
\hline $14 \beta$-pregnano & & & & & & & & & & & $x$ & \\
\hline 1-Docosanol (C22) & & & & & & 5,6 & & & & & & \\
\hline Docosano (C22) & & & & & & 13,8 & & & & & & \\
\hline Tricosano (C23) & & & & & & & & & & & & \\
\hline Tetracosano (C24) & & & & & & & & & & & $\mathrm{X}$ & \\
\hline $\begin{array}{l}\text { Heptacosano } \\
\text { (C27) }\end{array}$ & & & & & & & 4,1 & & & & & \\
\hline $\begin{array}{l}\text { (3ß)-colest-5-en-3- } \\
\text { ol o colesterol } \\
\text { (C27) }\end{array}$ & & & & & & & & & 1,5 & & & \\
\hline $\begin{array}{l}\text { 5ß-etenil-A- } \\
\text { norcolestan-3-ona }\end{array}$ & & & & 1,7 & & & & & & & & \\
\hline $\begin{array}{l}\text { 3,5-colestadien-7- } \\
\text { ona }\end{array}$ & & & & 5,5 & & & & & & & & \\
\hline 7-oxo-colesterol & & & & 2,3 & & & & & & & & \\
\hline$\beta$-Sitosterol (C29) & & & & & & & & & 8,5 & & & \\
\hline Escualeno (C30) & 15,7 & & 10,1 & & & & & & & & $\mathrm{X}$ & $\mathrm{X}$ \\
\hline $\begin{array}{l}\text { Amirina (alfa o } \\
\text { beta) }\end{array}$ & & & & & & & & & & & $X$ & \\
\hline Dotriacontano (32) & & & & & & & 6,4 & & & & & \\
\hline Ceras $^{5}$ & & & & & & & & & 1,2 & $X^{4}$ & & $x$ \\
\hline Indeterminados & 11,4 & 20,0 & 5,3 & 70,7 & 11,7 & 19,7 & 80,8 & 25,1 & 6,8 & & & \\
\hline
\end{tabular}

(Figura 3E'). Debido a esto, se lo define como un bifaz en proceso de manufactura de aprendiz, con una expectativa nula de uso. La ausencia de indicadores significativos de uso es consistente con esto, ya que el análisis de microfósiles únicamente ha arrojado en un muy bajo número, cuerpos discoidales birrefringentes indeterminados (Figura $3 \mathrm{~F}^{\prime}-\mathrm{G}^{\prime}$ ) 
que como en los casos anteriores, podrían proceder del uso de un percutor blando de madera, remitiendo así a la fase de manufactura.

La debilidad de las señales químicas y microfósiles en los bifaces estudiados podría explicarse en parte por la naturaleza de la materia prima sobre la que se manufacturaron estos instrumentos, muy lisa y con baja capacidad de captura. Esta situación ya ha sido señalada en oportunidades anteriores en que se analizaron artefactos líticos tallados en vulcanitas con texturas afaníticas o microporfídicas de QS3 y de otros sitios del área (Babot et al. 2013; Escola et al. 2013). Sin embargo, en esos casos, los conjuntos microfósiles, los lípidos y los análisis de desgaste por uso mostraron un grado mayor de diagnosis y también mayores abundancias que en el presente caso de estudio. Por consiguiente, la consideración conjunta de las evidencias de uso y los atributos tecnológicos y

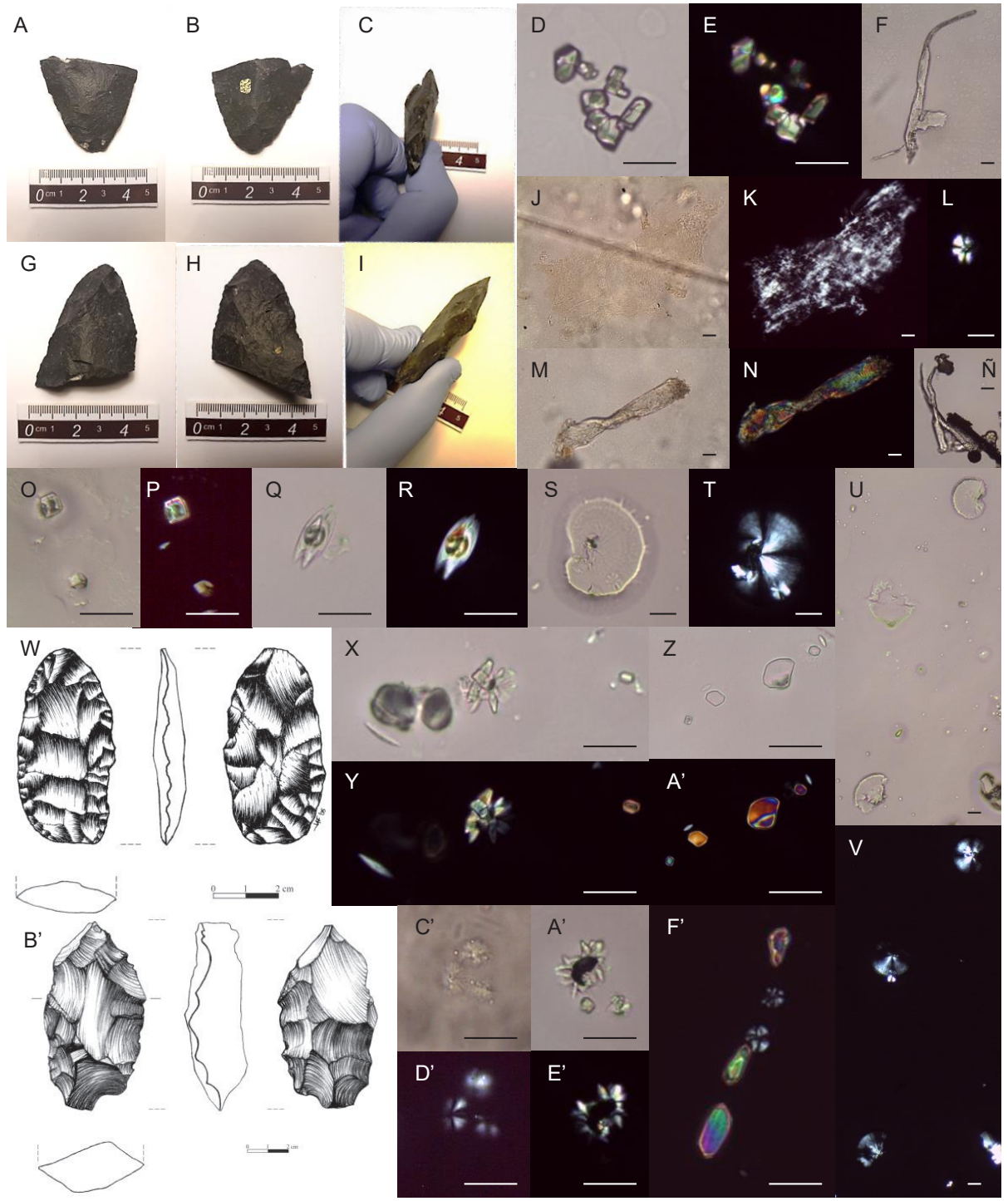

Figura 3. Bifaces del sitio QS3. A-F. Pieza No 28. A-C. Caras A y B y vista lateral. D-E. Cristales prismáticos de oxalato de calcio. F. Tricoma silicificado. G-V. Pieza N 632. G-I. Caras A y B y vista lateral. J-K. Tejido celulósico. L. Roseta de oxalato cálcico. M-N. Fibra. Ñ. Tricomas silicificados. O-P y Q-R. Cristales de oxalato de calcio prismáticos y naviculares, respectivamente. S-V. Cuerpos birrefringentes indeterminados. W- $D^{\prime}$. Pieza $N^{\circ}$ 593. W. Caras A y B, vista lateral y sección transversal. X-D'. Cristales prismáticos y naviculares, drusas y rosetas de oxalato de calcio. $E^{\prime}-G^{\prime}$ Pieza $N^{\circ} 316$. E'. Caras A y B, vista lateral y sección transversal. $F^{\prime}-G^{\prime}$. Cuerpos birrefringentes indeterminados.

Figure 3. Bifaces of the QS3 site. A-F. Artifact No. 28. A-C. Faces A and B and side view. OF. Prismatic crystals of calcium oxalate. F. Silicified trichome. G-V Artifact No 632. G-I. Faces $A$ and B and side view. J-K Cellulose tissue L. Rosette of calcium oxalate. $M-N$ Fiber. N Silicified trichome. O-P and Q-R. Prismatic and navicular calcium oxalate crystals, respectively. S-V. Indeterminate birefringent bodies. $W-D$ '. Artifact No. 593. W. Faces $A$ and $B$, side view and cross section. $X-D$ '. Prismatic and navicular crystals, druses and rosettes of calcium oxalate. $E^{\prime}-G$ 'Artifact No. 316. E'. Faces $A$ and B, side view and cross section. $F^{\prime}-G$ '. Indeterminate birefringent bodies. 
de destrezas diferenciales de los artesanos que habrían tallado los bifaces, más su incorporación en contextos de producción y mantenimiento del utillaje lítico en los niveles considerados del sitio, permite suponer que, en efecto, se trata de piezas en proceso de manufactura, mantenimiento o bien, con un uso acotado, según el caso. De este modo, se integrarían al resto de evidencias recuperadas en el sitio entre los 5500-4500 AP que remiten a un locus de residencia temporal en donde se condujeron prácticas artesanales referidas al instrumental en piedra y potencialmente, situaciones de enseñanza-aprendizaje (Hocsman 2007), con sus respectivos productos, desechos, insumos y herramientas. Estas implicaron gestualidades diversas, la utilización de diferentes materiales, la piedra, resinas, maderas no locales (caña chusquea y, potencialmente, sauce criollo), ataduras en tiento $y$, posiblemente, bolsas en fibra de palma, en torno al fuego, como fuente de luz y calor y también como un agente transformador (de resinas, probablemente madera y piedra) en estos procesos. Asimismo, estas prácticas involucraron conceptos de versatilidad para el caso de los bifaces, como se desprende de las piezas estudiadas, y de transformación, ya discutido para las puntas-cuchillos, además del criterio de uso manual o bien enmangado de esta clase de utillaje de procesamiento. La notable consistencia en estos aspectos a lo largo de los aproximadamente mil años reafirma el carácter perdurable del modo de ocupación de este locus y de las prácticas involucradas en él.

\section{Consideraciones Finales}

Si bien en nuestro país los abordajes integrales son de larga data, sobre la base de conjugar las perspectivas tecno-tipológicas y funcional de base microscópica (microhuellas), el desarrollo y aplicación de otras metodologías de análisis funcional, estudios contextuales y de saberes tradicionales, lleva a la posibilidad de profundizar en los alcances y capacidad interpretativa de las aproximaciones a los artefactos líticos. Al respecto, un punto crucial es cómo se articulan y combinan diferentes líneas de evidencia según su pertinencia para las preguntas que se formulan y los puntos de partida que guían los protocolos de análisis, como se ilustra a través de los casos de bifaces. Asimismo, es imprescindible la consideración no sólo de las potencialidades sino también de las limitaciones de cada línea, sin preconceptos, en contextos con o sin preservación de restos orgánicos (Babot et al. 2013; Mazzia et al. 2016). Finalmente, una aproximación de esta naturaleza cobra sentido al orientarse hacia la definición de la historia de vida artefactual y su inserción en las prácticas sociales.

San Miguel de Tucumán, 27 de Marzo de 2018.

\section{Agradecimientos}

Este trabajo se desarrolló en el marco de los Proyectos FONCYTPICT 1703, PIP-CONICET 577 y PIUNT 26/G605. Agradecemos a la Dra. R. Cattáneo la cortesía de facilitarnos información preliminar sobre análisis de huellas de uso en los bifaces de QS3.

\section{Bibliografía}

Álvarez, M.R. 2009. Diseño y función. Variabilidad instrumental en la costa Norte del Canal Beagle. En: Arqueología Argentina en los Inicios del Nuevo Siglo, editado por C. Gradin y F. Oliva, Tomo II, pp. 293-306. Escuela de Antropología, Facultad de Humanidades y Artes, Universidad Nacional de Rosario. Laborde Editor, Rosario.

Aschero, C.A. 1975. Ensayo para una clasificación morfológica de artefactos líticos aplicada a estudios tipológicos comparativos. Informe al CONICET. Manuscrito en posesión del autor.

Aschero, C.A. 1983. Ensayo para una clasificación morfológica de artefactos líticos aplicada a estudios tipológicos comparativos. Apéndices $A-C$. Revisión. Cátedra de Ergología y Tecnología (FFyL-UBA). Manuscrito en posesión del autor.

Aschero C.A., D. Elkin y E. Pintar 1991. Aprovechamiento de recursos faunísticos y producción lítica en el precerámico tardío. Un caso de estudio: Quebrada Seca 3 (Puna Meridional Argentina). En: Actas XII Congreso Nacional de Arqueología Chilena 2, pp. 101-114. Santiago de Chile.

Aschero, C.A. y S. Hocsman 2004. Revisando cuestiones tipológicas en torno a la clasificación de artefactos bifaciales. En: Temas de Arqueología. Análisis Lítico, compilado por M. Ramos, A. Acosta y D. Loponte, pp. 7-25. Universidad Nacional de Lujan, Lujan.

Aschero C.A., L. Manzi L, G. Gómez 1993/94. Producción lítica y uso del espacio en el nivel 2b4 de Quebrada Seca 3. Relaciones de la Sociedad Argentina de Antropología 19: 191-214.

Babot, M.P. 1999. "Un estudio de artefactos de molienda. Casos del Formativo". Tesina de grado, Facultad de Ciencias Naturales e Instituto Miguel Lillo, Universidad Nacional de Tucumán. San Miguel de Tucumán, Argentina.

Babot, M.P. 2004. "Tecnología y utilización de artefactos de molienda en el Noroeste prehispánico". Tesis de postgrado, Facultad de Ciencias Naturales e Instituto Miguel Lillo, Universidad Nacional de Tucumán San Miguel de Tucumán, Argentina.

Babot, M.P. 2010. Almidones y fitolitos: desentrañando el papel funcional de los artefactos de molienda arqueológicos. En: Arqueología argentina en los inicios de un nuevo siglo, compilado por F. Oliva, N. de Grandis, y J. Rodríguez, Tomo III, pp. 665-673. Laborde Editor, Rosario.

Babot, M.P., R.G. Cattáneo y S. Hocsman 2010. ¿Puntas de 
proyectil o cuchillos? Múltiples técnicas analíticas para una caracterización funcional de artefactos arqueológicos. En: La arqueometría en Argentina y Latinoamérica, editado por S. Bertolino, G.R. Cattaneo y A.D. Izeta, pp.127-134. Editorial de la Facultad de Filosofía y Humanidades, Universidad Nacional de Córdoba. Córdoba.

Babot, M.P., P.S. Escola y S. Hocsman 2008. Microfósiles y atributos tecno-tipológicos: Correlacionando raederas de módulo grandísimo con sus desechos de talla de mantenimiento en el Noroeste argentino. En: Matices interdisciplinarios en estudios fitolíticos y de otros microfósiles, editado por M.A. Korstanje y M.P. Babot, pp. 187-200. British Archaeological Reports (BAR) International Series 1870. Hedges. Oxford.

Babot, M.P., S. Hocsman y G.R. Cattáneo 2013. Assessing the life history of projectile points or knives from Middle Holocene of Southern Puna of Argentina. Quaternary International 287: 3-19.

Belén-Camacho, D.R., I. López, D. García, M. González, M.J. Moreno-Álvarez y C. Medina 2005. Evaluación fisicoquímica de la semilla y del aceite de corozo (Acrocomia aculeata Jacq.). Grasas y Aceites 56(4): 311-316.

Bruni, S.F. y V. Guglielmi 2014. Identification of archaeological triterpenic resins by the non-separative techniques FTIR and 13C NMR: the case of Pistacia resin (mastic) in comparison with frankincense. Spectrochimica Acta Parte A 121: 613-622.

Cattáneo, G.R. y A.M. Aguerre 2009. Estudios funcionales de artefactos líticos de Cueva de Las Manos, Río Pinturas, Santa Cruz, Argentina. Revista del Museo de Antropología 2: 3-22.

Cattáneo, G.R., M. Martinelli; A. Izeta, J.M. Caminoa y A. Robledo 2017. On wedges and bones: archaeological studies of use-wear and residue analysis from Late Holocene occupations in the Southern Pampean Hills (Alero Deodoro Roca, Córdoba, Argentina). Journal of Archaeological Science Reports 14: 275-288.

Duarte, M.R. y L. Chella 2013. Leaf and stem anatomical identification of creole willow: Salix humboldtiana Willd., Salicaceae. Visão Acadêmica 14(4): 5-15.

Escola, P.S., S. Hocsman y M.P. Babot 2013. Entre las residencias y los campos de cultivo. Aporte de los cuchillos/raederas de módulo grandísimo a la cuestión del laboreo agrícola en Antofagasta de la Sierra (Puna de Catamarca) durante el primer milenio d.C. Relaciones de la Sociedad Argentina de Antropología XXXVIII(1): 83-110.

Hocsman, S. 2007. Producción de bifaces y aprendices en el sitio Quebrada Seca 3- Antofagasta de la Sierra, Catamarca (5500-4500 ap). En: Producción y circulación prehispánicas de bienes en el sur andino, compilado por A.E. Nielsen, M.C. Rivolta, V. Seldes, M.M. Vázquez y P.H.
Mercolli, Vol. 2, pp. 55-82. Editorial Brujas. Córdoba.

Hocsman, S. 2009. Una propuesta de aproximación teórico-metodológica a conjuntos de artefactos líticos tallados. En Perspectivas Actuales en Arqueología Argentina, editado por R. Barberena, K. Borrazo y L. Borrero, pp. 271-302. Departamento de Investigaciones Prehistóricas y Arqueológicas, IMHICIHU, CONICET, Buenos Aires.

Hocsman, S. 2019. Las múltiples caras de los bifaces. Una propuesta de análisis multidimensional de sus historias de vida. Manuscrito en posesión del autor.

Landini, C., M. Bonomo, M. Leipus y G. Martínez, G. 2000. Forma y función de los instrumentos líticos del sitio Paso Otero 3 (pdo. de Necochea, pcia. de Buenos Aires, Argentina): un estudio comparativo. Espacio, Tiempo y Forma, Serie I, Prehistoria y Arqueología 13: 161-187.

Larrahondo, J.E. y T.R. Preston 1989. Control químico de la inversión de jugos de caña de azúcar para la alimentación animal. Livestock Research for Rural Development 1, Article \#6. http://www.Irrd.org//rrd1/1/lara.htm

Leipus, M. 2016. Variabilidad tecnomorfológica y funcional de las raederas en la Región Pampeana (Argentina). Revista de Antropología del Museo de Entre Ríos 2(2): 47-67.

Mansur, M.E. 1986. Microscopie du matériel lithique préhistorique. Traces d'utilisation, altérations naturelles, accidentelles et technologiques. Traces d'utilisation, altérations naturelles, accidentelles et technologiques. Exemples de Patagonie. Cahiers du Quaternaire $\mathrm{N}^{\circ}$ 9, Centre Régional de Publication de Bordeaux, Centre National de la Recherche Scientifique. Éditions du Centre National de la Recherche Scientifique, Paris.

Massigoge, A. y N. Pal 2011. Producción y uso de artefactos líticos en contextos cazadores-recolectores del Área Interserrana (Argentina): análisis integral de la diversidad tecno-morfológica y funcional. Revista Española de Antropología Americana 41(1): 51-73.

Mazzia, N., C. Weitzel y H. De Angelis 2016. De usos y recursos. Análisis funcional y de sustancias grasas mediante cromatografía gaseosas sobre artefactos líticos tallados. Revista del Museo de Antropología 9(2):37-50.

Rodríguez, M.F. 2013. Los grupos humanos y las plantas en la Puna meridional argentina: Arqueobotánica de Antofagasta de la Sierra. Intersecciones en Antropología 14: 315-339.

Sánchez Vizcaíno, A. y M.L. Cañabate Guerrero 1998. Indicadores químicos para la Arqueología. Servicio de Publicaciones e Intercambio Científico de la Universidad de Jaén, Colección Martínez de Mazas, Serie Estudios, Jaén. 